Bull. Austral. Math. Soc.

VOL. 43 (1991) [509-517]

\title{
RECURRENCE IN PERSISTENT DYNAMICAL SYSTEMS
}

\author{
Sung Kyo Choi, Chin-Ku Chu and Keon-Hee Lee
}

The purpose of this paper is to study the chain recurrent sets under persistent dynamical systems, and give a necessary condition for a persistent dynamical system to be topologically stable. Moreover we show that the various recurrent sets depend continuously on persistent dynamical systems.

The abstract theory of dynamical systems distinguished various recurrence properties such as periodicity, Poisson stability, nonwanderingness, chain recurrence, et cetera. The weakest property among them is the property of a point to be chain recurrent.

In [3], Hurley analysed the chain recurrent sets under topologically stable dynamical systems, and Lewowicz [5] introduced the concept of persistence of a dynamical system which is weaker than that of topological stability.

The purpose of this paper is to study the chain recurrent sets under persistent dynamical systems, and give a necessary condition for a persistent dynamical system to be topologically stable. Bronstein and Kopanskii [1] introduced the concepts of weakly nonwandering set and chain recurrent set for dispersive dynamical systems (or a dynamical system without uniqueness) and said that, in general, it remains unknown whether or not the weakly nonwandering set is equal to the chain recurrent set. (See Section 6 in [1]). We claim that for a dynamical system (with uniqueness) the weakly nonwandering set is properly contained in the chain recurrent set. Moreover, Ombach [7] showed that the various recurrence mappings (such as $\alpha, \omega, \Omega, C R$, etcetera) are continuous at $f$ if the system $f$ has the P.O.T.P. (pseudo orbit tracing property) and is expansive. Finally we prove that the various recurrence mappings are also continuous at $f$ if the system $f$ is persistent.

We consider homeomorphisms (or dynamical systems) acting on a compact metric space. Unless otherwise mentioned, we let $X$ denote a compact metric space with a metric $d$. Let $H(X)$ denote the collection of all homeomorphisms of $X$ to itself topologised by the $C^{0}$-metric

$$
d_{0}(f, g)=\sup \{d(f(x), g(x)): x \in X\}
$$

Received 28 June 1990

This research is supported by Korea Science and Engineering Foundation, 1988-1989.

Copyright Clearance Centre, Inc. Serial-fee code: 0004-9729/91 \$A2.00+0.00. 
$f \in H(X)$ is said to be topologically stable if for any $\varepsilon>0$ there exists $\delta(\varepsilon)>0$ such that if $d_{0}(f, g)<\delta$ then there exists a continuous map $h: X \rightarrow X$ satisfying $h g=f h$ and $d_{0}\left(h, 1_{X}\right)<\varepsilon$, where $1_{X}$ is the identity map on $X$. The map $h$ is called the semiconjugacy from $g$ to $f$. We say that $f \in H(X)$ is exponsive if there exists $e(f)>0$ such that if $d\left(f^{n}(x), f^{n}(y)\right) \leqslant e$ for every $n \in Z$, then $x=y$. Such numbers $e(f)$ are called expansive constants. $f \in H(X)$ is called $\alpha$ (or $\beta$ )-persistent if for any $\varepsilon>0$ there exists $\delta(\varepsilon)>0$ such that if $d_{0}(f, g)<\delta$ and $x \in X$, then there is $y \in X$ satisfying

$$
d\left(f^{n}(y), g^{n}(x)\right)<\varepsilon\left(\text { or } d\left(f^{n}(x), g^{n}(y)\right)<\varepsilon\right),
$$

respectively, for all $n \in Z$.

Throughout this paper, it will be noted that a dynamical system means a discrete dynamical system induced by a homeomorphism on $X$, and a persistent dynamical system means both $\alpha$ and $\beta$-persistent dynamical system.

LEMma 1. A topologically stable dynamical system is persistent.

PROOF: It is straightforward.

The following theorem gives a necessary condition for a persistent dynamical system to be topologically stable.

THEOREM 2. A persistent dynamical system is topologically stable if it is expansive.

Proof: Let $f \in H(X)$ be persistent, and let $e(f)$ be an expansive constant for $f$. Choose $\varepsilon>0$ satisfying $\varepsilon<e(f) / 4$. Given $\varepsilon>0$, there exists $\delta>0$ such that if $d_{0}(f, g)<\delta$, then for any $x \in X$, there is $y \in X$ satisfying

$$
d\left(f^{n}(y), g^{n}(x)\right)<\varepsilon / 2
$$

for all $n \in Z$. Define a map $h: X \rightarrow X$ by $h(x)=y$, where $y$ is an element in $X$ chosen by the property of persistence of $f$ as above. Then the map $h$ is well-defined. In fact, let $z$ be another element in $X$ such that $d\left(f^{n}(z), g^{n}(x)\right)<\varepsilon / 2$ for all $n \in Z$. Then we have $d\left(f^{n}(y), f^{n}(z)\right)<\varepsilon$ for all $n \in Z$. Since $f$ is expansive, we get $y=z$.

Now we show that the map $h$ is continuous. Put $h(x)=y$ and $h\left(x^{\prime}\right)=y^{\prime}$, and let $\lambda>0$ be given. Since $f$ is expansive, we can choose $N$ such that if $d\left(f^{n}(y), f^{n}\left(y^{\prime}\right)\right) \leqslant$ $e(f)$ for all $-N \leqslant n \leqslant N$ then $d\left(y, y^{\prime}\right)<\lambda$. Suppose not. Then, for each $N \geqslant 1$, there exists $a_{N}, b_{N} \in X$ such that

$$
d\left(f^{n}\left(a_{N}\right), f^{n}\left(b_{N}\right)\right) \leqslant e(f) \text { and } d\left(a_{N}, b_{N}\right) \geqslant \lambda
$$

for all $-N \leqslant n \leqslant N$. Consider the sequences $\left\{a_{N}\right\}$ and $\left\{b_{N}\right\}$. Since $X$ is compact, we have $a_{N} \rightarrow a$ and $b_{N} \rightarrow b$ in $X$. Then we get $d(a, b) \geqslant \lambda$ and $d\left(f^{n}(a), f^{n}(b)\right) \leqslant e(f)$, 
for all $n \in Z$. This contracts the expansiveness of $f$. Since $g: X \rightarrow X$ is continuous, given $N$, there exists $\eta>0$ such that if $d\left(x, x^{\prime}\right)<\eta$ then $d\left(g^{n}(x), g^{n}\left(x^{\prime}\right)\right)<e(f) / 2$ for all $-N \leqslant n \leqslant N$. Consequently we have

$$
\begin{aligned}
d\left(f^{n}(y), f^{n}\left(y^{\prime}\right)\right) \leqslant & d\left(f^{n}(y), g^{n}(x)\right)+d\left(g^{n}(x), g^{n}\left(x^{\prime}\right)\right) \\
& +d\left(g^{n}\left(x^{\prime}\right), f^{n}\left(y^{\prime}\right)\right)<e(f),
\end{aligned}
$$

if $d\left(x, x^{\prime}\right)<\eta$ and $-N \leqslant n \leqslant N$. Thus we obtain $d\left(y, y^{\prime}\right)=d\left(h(x), h\left(x^{\prime}\right)\right)<\lambda$. By now, we have shown that for any $\lambda>0$ there exists $\eta>0$ such that if $d\left(x, x^{\prime}\right)<\eta$ then $d\left(h(x), h\left(x^{\prime}\right)\right)<\lambda$, that is, $h$ is continuous. Moreover, the surjectivity of $h$ can be derived from the fact that $f$ is $\beta$-persistent.

To show that $h g=f h$ on $X$, choose $x \in X$. Then there exists $y \in X$ satisfying $d\left(f^{n+1}(y), g^{n+1}(x)\right)<\varepsilon / 2$ for all $n \in Z$. Thus we get $h(g(x))=f(y)=f(h(x))$, by the definition of $h$. Hence we have $h g=f h$ on $X$. This completes the proof of the theorem.

We say that $f, g \in B(X)$ are topologically conjugate if there exists $h \in B(X)$ satisfying $h g=f h$, and the homeomorphism $h$ is called topological conjugacy between $f$ and $g$.

In the following theorem, we see that the $\alpha$ (or $\beta$ )-persistence is invariant under a topological conjugacy.

THEOREM 3. Any dynamical system which is topologically conjugate to an $\alpha$ (or $\beta$ )-persistent dynamical system is $\alpha$ (or $\beta$ )-persistent, respectively.

Proof: Suppose that an $\alpha$-persistent dynamical system $f$ is topologically conjugate to a dynamical system $g$, and let $h$ be a topological conjugacy between $f$ and $g$. Let $\varepsilon>0$ be given, and choose $0<\varepsilon^{\prime}<\varepsilon$ such that if $d(a, b)<\varepsilon^{\prime}$ then $d\left(h^{-1}(a), h^{-1}(b)\right)<\varepsilon$ for $a, b \in X$. Since $f$ is $\alpha$-persistent, given $\varepsilon^{\prime}>0$, there exists $\delta^{\prime}>0$ such that if $d_{0}\left(f, f_{0}\right)<\delta^{\prime}$ then for any $x \in X$ there is $y \in X$ satisfying $d\left(f^{n}(y), f_{0}^{n}(x)\right)<\varepsilon^{\prime}$ for all $n \in Z$. Given $\delta^{\prime}>0$, choose $0<\delta<\delta^{\prime}$ such that if $d(a, b)<\delta$ then $d(h(a), h(b))<\delta^{\prime}$. Let $g_{0} \in H(X)$ be such that $d_{0}\left(g, g_{0}\right)<\delta$, and put $f_{0}=h g_{0} h^{-1}$. Then we have

$$
d\left(h(g(x)), h\left(g_{0}(x)\right)\right)=d\left(f(h(x)), f_{0}(h(x))\right)<\delta^{\prime}
$$

for any $x \in X$, and so $d_{0}\left(f, f_{0}\right)<\delta^{\prime}$. Since $f$ is $\alpha$-persistent, given $h(x)$, there exists $h(y) \in X$ such that

$$
d\left(f^{n}(h(y)), f_{0}^{n}(h(x))\right)=d\left(h\left(g^{n}(y)\right), h\left(g_{0}^{n}(x)\right)\right)<\varepsilon^{\prime}
$$

for all $n \in Z$. Thus we have $d\left(g^{n}(y), g_{0}^{n}(x)\right)<\varepsilon$ for all $n \in Z$. This means that $g$ is $\alpha$-persistent. 
Similarly we can show that any dynamical system which is topologically conjugate to a $\beta$-persistent dynamical system is also $\beta$-persistent.

Let $f \in H(X)$. A point $x \in X$ is said to be periodic if there exists $n \geqslant 1$ satisfying $f^{n}(x)=x$. The set of all periodic points of $f$ will be denoted by $\operatorname{Per}(f)$. A point $x \in X$ is called nonwandering if for any neighbourhood $U$ of $x$, there exists $n \geqslant 1$ such that $f^{n}(U) \cap U \neq 0$. We denote by $\Omega(f)$ the set of all nonwandering points of $f$. Let $x$ and $y$ be any points in $X$, and let $\varepsilon>0$ be an arbitrary number. A finite sequence $\left\{x_{i}\right\}_{i=0}^{n}$ in $X$ is called an $\varepsilon$-chain from $x$ to $y$ provided that

$$
x_{0}=x, x_{n}=y \text { and } d\left(f\left(x_{i}\right), x_{i+1}\right)<\varepsilon,
$$

for $i=0,1, \cdots, n-1$. We say that $x$ is chain equivalent to $y$ if for any $\varepsilon>0$ there exist two $\varepsilon$-chains: from $x$ to $y$, and from $y$ to $x$. A point $x \in X$ is called chain recurrent if it is chain equivalent to itself. We denote by $C R(f)$ the set of all chain recurrent points of $f$. There is a natural equivalence relation defined on $C R(f): x \sim y$ if and only if $x$ is chain equivalent to $y$. Equivalence classes under this equivalence relation are called chain components of $f$.

A basic problem is to determine when a chain recurrent point is approximated by periodic points of $f$, or more generally to determine if each $\varepsilon$-chain can be approximated by an actual orbit of $f$. Here we claim that this problem can be done for $\alpha$-persistent dynamical systems on topological manifolds. To show this we need a lemma given in [6].

LEMMA 4. Let $X$ be a compact manifold of $\operatorname{dim} \geqslant 2$ with metric $d$, and let $\varepsilon>0$ be arbitrary. Then there exists $\delta(\varepsilon)>0$ such that if $\left\{\left(x_{1}, y_{1}\right), \cdots,\left(x_{n}, y_{n}\right)\right\}$ is a finite set of points in $X \times X$ satisfying:

(i) for each $i=1, \cdots, n, d\left(x_{i}, y_{i}\right)<\delta$; and

(ii) if $i \neq j$, then $x_{i} \neq x_{j}$ and $y_{i} \neq y_{j}$;

then there is $h \in H(X)$ with $d_{0}\left(h, 1_{X}\right)<\varepsilon$ and $h\left(x_{i}\right)=y_{i}$ for $i=1, \cdots, n$.

TheOREM 5. Let $X$ be a compact manifold, and let $f \in H(X)$ be $\alpha$-persistent. Then the set of all periodic points of $f$ is dense in $C R(f)$.

Proof: If $X$ is one-dimensional then the proof is clear. So, we may assume that the dimension of $X$ is larger than 1 . Let $\varepsilon>0$ be arbitrary. Then we select $\delta_{1}(\varepsilon)>0$ as in Lemma 4. Since $f$ is $\alpha$-persistent, there exists $\delta_{2}\left(\delta_{1}\right)>0$ such that if $d_{0}(f, g)<\delta_{2}$ and $x \in X$, then $d\left(f^{n}(y), g^{n}(x)\right)<\delta_{1}$ for some $y \in X$ and all $n \in Z$. Given $\delta_{2}>0$, we choose $\delta_{3}\left(\delta_{2}\right)>0$ as in Lemma 4. Let $x \in C R(f)$, and let $\left\{x_{0}, \cdots, x_{m}\right\}$ be a $\delta_{3}$-chain from $x$ to $x$. Then the set $\left\{\left(f\left(x_{0}\right), x_{1}\right), \cdots,\left(f\left(x_{m-1}\right), x_{m}\right)\right\}$ satisfies the hypothesis of Lemma 4. Thus there exists $h \in H(X)$ such that

$$
d_{0}\left(h, 1_{X}\right)<\delta_{2} \text { and } h\left(f\left(x_{i}\right)\right)=x_{i+1},
$$


for $i=0,1, \cdots, m-1$. Put $g=h f$. Then we have $d_{0}(f, g)<\delta_{2}$ and $g^{m}(x)=x$. Hence there is $y \in X$ satisfying $d\left(g^{n}(x), f^{n}(y)\right)<\delta_{1}$ for all $n \in Z$. Consider the set $\left\{(x, y),(g(x), f(y)), \cdots,\left(g^{m}(x), f^{m}(y)\right)\right\}$ in $X \times X$ satisfying the hypothesis of Lemma 4. Then we have $h^{\prime} \in H(X)$ such that

$$
d_{0}\left(h^{\prime}, 1_{X}\right)<\varepsilon \text { and } h^{\prime}\left(g^{i}(x)\right)=f^{i}(y)
$$

for $i=0,1, \cdots, m$. In particular, we have

$$
f^{m}(y)=h^{\prime}\left(g^{m}(x)\right)=h^{\prime}(x)=y .
$$

This means that $B(x, \varepsilon) \cap \operatorname{Per}(f) \neq \emptyset$, and so completes the proof.

In [3], Hurley showed that if $f$ is a topologically stable diffeomorphism on a smooth compact Riemannian manifold $X$ then each chain component of $f$ contains a dense orbit. Moreover, he claimed that if $f$ is topologically stable, $X$ is connected and $C R(f)$ has interior then $C R(f)=X$. We extend these results to $\alpha$-persistent homeomorphisms on a compact manifold.

THEOREM 6. Let $X$ be a compact manifold, and let $f \in H(X)$ be $\alpha$-persistent. Then each chain component of $f$ contains a dense orbit.

Proof: If $X$ is one-dimensional this is clear. Hence we may assume $\operatorname{dim} X \geqslant 2$. Let $F$ be a chain component in $C R(f)$, and let $U$ and $V$ be any nonempty open sets in $F$. For any $x \in U$ and $y \in V$, we choose $\varepsilon>0$ such that $B(x, \varepsilon) \subset U$ and $B(y, \varepsilon) \subset V$. Since $f$ is $\alpha$-persistent, there exists $\delta(\varepsilon)>0$ such that if $d_{0}(f, g)<\delta$ then $d\left(f^{n}(z), g^{n}(x)\right)<\varepsilon$ for some $z \in X$ and all $n \in Z$. Given $\delta>0$, we select $\delta^{\prime}(\delta)$ as in Lemma 4. Since $x, y \in F$, we can choose a $\delta^{\prime}$-chain $\left\{x_{0}, \cdots, x_{m}\right\}$ from $x$ to $y$. Then there exists $h \in B(X)$ such that

$$
d_{0}\left(h, 1_{X}\right)<\delta \text { and } h\left(f\left(x_{i}\right)\right)=x_{i+1}
$$

for $i=0,1, \cdots, m-1$. If we let $g=h f$, then there is $z \in X$ satisfying $d\left(f^{n}(z), g^{n}(x)\right)<\varepsilon$ for all $n \in Z$. In particular, we have $d(x, z)<\varepsilon$ and $d\left(f^{m}(z), g^{m}(x)\right)<\varepsilon$. Since $g^{m}(x)=y$, we get $f^{m}(z) \in f^{m}(U) \cap V \neq \emptyset$. This implies that $F$ has a dense orbit.

THEOREM 7. If $C R(f)$ is connected, then $X$ is the chain component of any point in $X$. In particular, we have $C R(f)=X$.

Proof: Let $\varepsilon>0$ be fixed and $x \in X$. Let $F(x, \varepsilon)=\{y \in C R(f)$ : there exist two $\varepsilon$-chains, from $x$ to $y$ and from $y$ to $x$ \}.

First we show that $F(x, \varepsilon)$ is open and closed in $C R(f)$. If $y \in F(x, \varepsilon)$, then there exist two $\varepsilon$-chains: $\left\{x_{i}\right\}_{i=1}^{m}$ from $x$ to $y$, and $\left\{y_{j}\right\}_{j=1}^{n}$ from $y$ to $x$. Since 
$f(y) \in B\left(y_{1}, \varepsilon\right)$, we can choose $\delta_{1}>0$ such that $B\left(f(y), \delta_{1}\right) \subset B\left(y_{1}, \varepsilon\right)$. If we use the continuity of $f$, we can select $\delta_{2}>0$ satisfying

$$
f\left(B\left(y, \delta_{2}\right)\right) \subset B\left(f(y), \delta_{1}\right) \subset B\left(y_{1}, \varepsilon\right) .
$$

Let $U=B(y, \varepsilon) \cap B\left(f\left(x_{m-1}\right), \varepsilon\right)$. Then $U$ is an open neighbourhood of $y$ contained in $F(x, \varepsilon)$. In fact, for any $z \in U$, the sequence $\left\{x_{0}, x_{1}, \cdots, x_{m-1}, z\right\}$ is an $\varepsilon$-chain from $x$ to $z$, and the sequence $\left\{z, y_{1}, \cdots, y_{n}\right\}$ is an $\varepsilon$-chain from $z$ to $x$. Thus we have $U \subset F(x, \varepsilon)$. This shows that $F(x, \varepsilon)$ is open in $C R(f)$. To show that $F(x, \varepsilon)$ is closed in $C R(f)$, we choose a sequence $\left\{x_{i}\right\}$ in $F(x, \varepsilon)$ which converges to $x^{0} \in X$. Then there exists $n \in N$ such that $d\left(x^{i}, x^{0}\right)<\varepsilon / 2$ for all $i \geqslant n$. Since $x^{n} \in F(x, \varepsilon) \subseteq C R(f)$, we can choose an $\varepsilon / 2$-chain $\left\{x_{0}^{n}, x_{1}^{n}, \cdots, x_{k}^{n}\right\}$ from $x^{n}$ to $x^{n}$. Consider the sequence $\left\{x_{0}^{n}, x_{1}^{n}, \cdots, x_{k-1}^{n}, x^{0}\right\}$. Then it is an $\varepsilon$-chain from $x^{n}$ to $x^{0}$. Similarly we can construct an $\varepsilon$-chain from $x^{0}$ to $x^{n}$. This means that $F(x, \varepsilon)$ is closed in $C R(f)$.

Next we show that $X$ is the chain component of $x$. Since $C R(f)$ is connected, we have $C R(f)=F(x, \varepsilon)$. Let $F(x)$ be the chain component of $x$. Since $F(x)=$ $\bigcap_{\varepsilon 0} F(x, \varepsilon)$, the proof is completed by showing that $C R(f)=X$. So, we suppose that $C R(f) \neq X$. Then there exists $y \in X$ with $y \notin C R(f)$. Let $\omega_{f}(y)=\{Z \in X$ : $f^{n_{i}}(y) \rightarrow z$ for some $\left.n_{i} \rightarrow-\infty\right\}$, and let $z \in \omega_{f}(y)$. Then we have

$$
z \in \omega_{f}(y) \subset \Omega(f) \subset C R(f) .
$$

Since $C R(f)=F(x, \varepsilon)$, we can choose two $\varepsilon$-chains: $\left\{x_{i}\right\}_{i=0}^{k}$ from $x$ to $z$, and $\left\{z_{i}\right\}_{i=0}^{p}$ from $z$ to $x$. Using the continuity of $f$, we can select $m<0$ such that $d\left(f(z), f^{m+1}(y)\right)<\varepsilon$. Then the sequence

$$
\left\{x_{0}, x_{1}, \cdots, x_{k}, f^{m+1}(y), \cdots, f^{-1}(y), y\right\}
$$

is an $\varepsilon$-chain from $x$ to $y$. Similarly we can construct an $\varepsilon$-chain from $y$ to $x$. Thus we have $y \in F(x, \varepsilon)$. This contradicts the fact that $y \notin C R(f)$, and so completes the proof.

Bronstein and Kopanskii introduced the notions of weakly nonwandering set and chain recurrent set for a dispersive dynamical system (or a dynamical system without uniqueness) on a compact metric space, and said that, in general, it remains unknown whether or not the weakly nonwandering set is equal to the chain recurrent set (see Section 6 in [1]). Clearly, a dynamical system (with uniqueness) on a compact metric space is also a dispersive dynamical system.

Similarly we introduce the concept of weakly nonwandering set of a dynamical system on a compact metric space. 
A point $x \in X$ is called weakly nonwandering (or weakly periodic) for $f \in H(X)$ if for any $\varepsilon>0$ there exists $g \in H(X)$ such that $d_{0}(f, g)<\varepsilon$ and $x$ is nonwandering (or periodic) for $g$, respectively. The set of all weakly nonwandering (or weakly periodic) points for $f$ will be denoted by $\Omega_{w}(f)$ (or $P_{w}(f)$ ), respectively. It is easy to show that $\Omega_{w}(f)$ is closed and $\Omega(f) \subset \Omega_{w}(f)$.

In the following example, we show that for a dynamical system $f$ (with uniqueness), the set $\Omega_{w}(r)$ is not equal to $C R(f)$.

EXAmple 8: Let $X$ be a subset of $\mathrm{R}^{2}$ given by $X=S^{1} \cup L$, where $S^{1}=\{(x, y) \in$ $\left.\mathbf{R}^{2}: x^{2}+y^{2}=1\right\}$ and $L=\left\{(x, y) \in R^{2}:-1<x<1\right.$ and $\left.y=0\right\}$. Then we define a homeomorphism $f_{1}$ on $S^{1}$ satisfying: $(-1,0)$ and $(1,0)$ are fixed points of $f_{1}$, and for any $(x, y) \in S^{1}-\{(-1,0),(1,0)\}$ the first coordinate of $f_{1}(x, y)$ is larger than $x$. Also we define a homeomorphism $f_{2}$ on $L$ satisfying: for any $(x, y) \in L$ the first coordinate of $f_{2}(x, y)$ is less than $x$. Then we can define a homeomorphism $f$ on the compact metric space $X$ such that $\left.f\right|_{S^{1}}=f_{1}$ and $\left.f\right|_{L}=f_{2}$. For the homeomorphism $f$, we have $C R(f)=X$ and $\Omega_{w}(f)=\{(-1,0),(1,0)\}$.

Theorem 9. For any $f \in H(X)$, the set $\Omega_{w}(f)$ is contained in $C R(f)$, and the converse holds if $X$ is a compact manifold.

Proof: Let $x \in \Omega_{w}(f)$, and let $\varepsilon>0$ be arbitrary. Then we have $g \in H(X)$ such that $d_{0}(f, g)<\varepsilon / 4$ and $x \in \Omega(g)$. Choose $\delta<\varepsilon / 4$ such that if $d(x, y)<\delta$ then $d(f(x), g(x))<\varepsilon / 4$ and $d(g(x), g(y))<\varepsilon / 4$. Since $x \in \Omega(g)$, there exists $n \geqslant 1$ satisfying $g^{n}(B(x, \delta)) \cap B(x, \delta) \neq \emptyset$. If $n=1$ then the sequence $\{x, x\}$ is an $\varepsilon$-chain for $f$. In fact, if we choose $y \in B(x, \delta)$ with $g(y) \in B(x, \delta)$, then we get

$$
\begin{aligned}
d(f(x), x) \leqslant & d(f(x), g(x))+d(g(x), g(y)) \\
& +d(g(y), x)<\varepsilon .
\end{aligned}
$$

If $n>1$ then there exists $y \in B(x, \delta)$ with $g^{n}(y) \in B(x, \delta)$. Then the sequence $\left\{x, g(y), \cdots, g^{n-1}(y), x\right\}$ is an $\varepsilon$-chain from $x$ to $x$. This shows that $x \in C R(f)$.

The converse can be derived in the proof of Theorem 6 .

For any $f \in H(X)$ and any $x \in X$, we let $\alpha_{f}(x)=\left\{y \in X: f^{n_{i}}(x) \rightarrow\right.$ $y$ for some $\left.n_{i} \rightarrow \infty\right\}$ and $\omega_{f}(x)=\left\{y \in X: f^{n_{i}}(x) \rightarrow y\right.$ for some $\left.n_{i} \rightarrow-\infty\right\}$. Then the sets $\alpha(f)=\bigcup_{x \in X} \alpha_{f}(x)$ and $\omega(f)=\bigcup_{x \in X} \omega_{f}(x)$ are called the negative and positive limit sets for $f$, respectively. Let us consider the metric space $K(X)=\{A \subset X: A$ is closed $\}$ with the Hausdorff metric $\rho$

$$
\rho(A, B)=\max \left\{\sup _{a \in A} d(a, B), \sup _{b \in B} d(A, b)\right\} .
$$

Let $\alpha, \omega, \Omega, \Omega_{w}, C R$ denote mappings $H(X) \rightarrow K(X)$ sending $f$ to $\overline{\alpha(f)}, \overline{\omega(f)}$, $\Omega(f), \Omega_{w}(f), C R(f)$, respectively. 
In [7], Ombach proved that the mappings defined as above are continuous at $f$ if $f$ has the P.O.T.P. and is expansive. It is well-known that a dynamical system which is both expansive and possesses the P.O.T.P. is topologically stable. By Lemma 1, a topologically stable dynamical system is persistent. Finally, we show that the mappings defined as above are also continuous at $f$ if $f$ is persistent. To show this we need the concept of upper and lower semi-continuity.

Let $Y$ be a topological space. A mapping $F: Y \rightarrow K(X)$ is upper (or lower) semi-continuous at $y \in Y$, if for any $\varepsilon>0$ there exists a neighbourhood $U$ of $y$ such that for any $z \in U$ we have

$$
F(z) \subset B_{c}(F(y)) \text { (or } F(y) \subset B_{c}(F(z)) \text { ), }
$$

respectively, where $B_{\varepsilon}(A)=\{y \in X: d(x, y)<\varepsilon$, for some $x \in A\}$.

Lemma 10. A mapping $F: Y \rightarrow F(X)$ is continuous at $y \in Y$ if and only if $F$ is upper and lower semi-continuous at $y$.

THEOREM 11. Let $X$ be a compact manifold, and let $f \in H(X)$ be persistent. Then the mappings $\alpha, \omega, \Omega, \Omega_{w}, C R$ are continuous at $f$.

Proof: Let $\varepsilon>0$ be arbitrary. Since $f$ is persistent, we can choose $\delta_{1}>0$ such that if $d_{0}(f, g)<\delta_{1}$ and $x \in X$, then $d\left(f^{n}(x), g^{n}(y)\right)<\varepsilon / 3$ for some $y \in X$ and all $n \in Z$. Let $g \in H(X)$ be such that $d_{0}(f, g)<\delta_{1}$. Then for any $x \in X$, there exists $y \in X$ satisfying $d\left(f^{n}(x), g^{n}(y)\right)<\varepsilon / 3$ for all $n \in Z$. This means that

$$
\begin{aligned}
& \omega_{f}(x) \subset B_{\varepsilon / 2}\left(\omega_{g}(y)\right) \subset B_{\varepsilon / 2}(\overline{\omega(g)}), \text { and } \\
& \alpha_{f}(x) \subset B_{\varepsilon / 2}\left(\alpha_{g}(y)\right) \subset B_{\varepsilon / 2}(\overline{\alpha(g)}) .
\end{aligned}
$$

Thus we have

$$
\overline{\omega(f)} \subset B_{e}(\overline{\omega(g)}) \text { and } \overline{\alpha(f)} \subset B_{e}(\overline{\alpha(g)}) .
$$

Since the mapping $C R: H(X) \rightarrow K(X)$ is upper semi-continuous at $f$, by Corollary 3(a) in [2], we can choose $\delta_{2}>0$ such that if $d_{0}(f, g)<\delta_{2}$ then $C R(g) \subset B_{\varepsilon}(C R(f))$. Let $\delta=\min \left(\delta_{1}, \delta_{2}\right)$, and let $g \in H(X)$ be such that $d_{0}(f, g)<\delta$. Since $C R(f)=$ $\overline{\operatorname{Per}(f)}$ by Theorem 5 , we have

$$
C R(f)=\overline{\operatorname{Per}(f)} \subset \overline{\omega(f)} \subset B_{e}(\overline{\omega(g)}) \subset B_{\varepsilon}(C \dot{R}(g)) .
$$

This implies that all considered mappings are lower semi-continuous at $f$. On the other hand, we have

$$
\overline{\alpha(g)} \cup \overline{\omega(g)} \subset C R(g) \subset B_{\varepsilon}(C R(f))=B_{\varepsilon}(\overline{\operatorname{Per}(f)})
$$


This means that all considered mappings are upper semi-continuous at $f$. By Lemma 10 , we complete the proof.

REMARKS 12: We know that $K(X)$ is again a compact metric space. Let $K(K(X))$ denote the space of all closed non-empty subsets of $K(X)$ with the Hausdorff metric $\bar{\rho}$. Then for each $f \in \boldsymbol{H}(X)$ and $x \in X$, the set $\overline{O(f, x)}=\overline{\left\{f^{n}(x): n \in Z\right\}}$ may be interpreted as a point of $K(X)$ and the set $\overline{O(f)}=\overline{\{\overline{O(f, x)}: x \in X\}}$ can be interpreted as a point of $K(K(X))$. If we let $O$ denote a mapping from $H(X)$ into $K(K(X))$, sending $f \in H(X)$ to $\overline{O(f)}$, then we can easily see that the mapping $O$ is continuous whenever $f$ is persistent.

\section{REFERENCES}

[1] I.U. Bronstein and A.Ya. Kopanskii, 'Chain recurrence in dynamical systems without uniqueness', Nonlinear Anal. 12 (1988), 147-154.

[2] M. Hurley, 'Bifurcation and chain recurrence', Ergodic Theory Dynamical Systems 3 (1983), 231-240.

[3] M. Hurley, 'Consequences of topological stability', J. Differential Equations 54 (1984), 60-72.

[4] K.H. Lee, 'Recurrence in Lipschitz stable flows', Bull. Austral. Math. Soc. 38 (1988), 197-202.

[5] J. Lewowicz, 'Persistence in expansive systems', Ergodic Theory Dynamical Systems 3 (1983), 567-578.

[6] Z. Nitecki and M. Shub, 'Filtrations, decompositions and explosions', Amer. J. Math. 97 (1976), 1029-1047.

[7] J. Ombach, 'Consequences of the P.O.T.P. and expansiveness', J. Austral. Math. Soc. Ser. A 43 (1987), 301-313.

Korea 